\title{
Existence of weak solutions for a class of quasilinear elliptic systems
}

\section{Zeng-Qi Ou* and Chun Li}

\section{"Correspondence:}

ouzengq707@sina.com

School of Mathematics and

Statistics, Southwest University,

Chongqing, 400715, People's

Republic of China

\begin{abstract}
Existence of weak solutions for a class of nonlinear elliptic systems is obtained under the certain Landesman-Lazer-type conditions by variational method.
\end{abstract}

Keywords: elliptic systems; Landesman-Lazer-type condition; (PS) condition

\section{Introduction and main results}

In this paper, we consider the existence of weak solutions for the following gradient elliptic systems:

$$
\begin{cases}-\triangle_{p} u=\lambda_{1} a(x)|u|^{p-2} u+\lambda_{1} \frac{b(x)}{\beta+1}|u|^{\alpha}|v|^{\beta} v+F_{u}(x, u, v)-h_{1}(x) & \text { in } \Omega \\ -\triangle_{p} v=\lambda_{1} c(x)|v|^{p-2} v+\lambda_{1} \frac{b(x)}{\alpha+1}|u|^{\alpha}|v|^{\beta} u+F_{v}(x, u, v)-h_{2}(x) & \text { in } \Omega \\ u=v=0 & \text { on } \partial \Omega\end{cases}
$$

where $\Omega \subset R^{N}(N \geq 3)$ is a bounded smooth domain, $\triangle_{p} u=\operatorname{div}\left(|\nabla u|^{p-2} \nabla u\right)$ denotes the $p$-Laplacian, $2 \leq p<N$ and $\alpha \geq 0, \beta \geq 0$ satisfy

$$
\alpha+\beta+2=p
$$

$F \in C^{1}\left(\bar{\Omega} \times R^{2}, R\right)$ and $F_{s}(x, s, t)$ designates the partial derivative of $F$ with respect to $s$ and $h_{1}, h_{2} \in L^{q}(\Omega)(q=p /(p-1))$. The coefficient functions $a, b, c \in C(\Omega) \cap L^{\infty}(\Omega)$ satisfy one of the following conditions:

(A1) $a^{+} \neq 0$, where $a^{+}(x):=\max \{a(x), 0\}$

(A2) $c^{+} \neq 0$;

(A3) $a=c=0$ and $b^{+} \neq 0$.

Let $W$ be the product space $W_{0}^{1, p}(\Omega) \times W_{0}^{1, p}(\Omega)$ equipped with the norm $\|(u, v)\|=$ $\left(\|u\|^{p}+\|v\|^{p}\right)^{1 / p}$ for all $(u, v) \in W$, where $\|u\|=\left(\int_{\Omega}|\nabla u|^{p} d x\right)^{1 / p}$ for any $u \in W_{0}^{1, p}(\Omega)$. The embedding $W_{0}^{1, p}(\Omega) \hookrightarrow L^{p}(\Omega)$ is continuous and there exists a positive constant $C$ such that

$$
\|u\|_{L^{p}} \leq C\|u\| \quad \text { for all } u \in W_{0}^{1, p}(\Omega)
$$

where $\|\cdot\|_{L^{p}}$ denotes the norm of $L^{p}(\Omega)$.

(c) $2015 \mathrm{Ou}$ and Li. This article is distributed under the terms of the Creative Commons Attribution 4.0 International License (http://creativecommons.org/licenses/by/4.0/), which permits unrestricted use, distribution, and reproduction in any medium, provided you give appropriate credit to the original author(s) and the source, provide a link to the Creative Commons license, and indicate if changes were made. 
Consider the following nonlinear eigenvalue problem with weights:

$$
\begin{cases}-\triangle_{p} u=\lambda a(x)|u|^{p-2} u+\lambda \frac{b(x)}{\beta+1}|u|^{\alpha}|v|^{\beta} v & \text { in } \Omega, \\ -\triangle_{p} v=\lambda c(x)|v|^{p-2} v+\lambda \frac{b(x)}{\alpha+1}|u|^{\alpha}|v|^{\beta} u & \text { in } \Omega, \\ u=v=0 & \text { on } \partial \Omega .\end{cases}
$$

If one of the conditions (A1)-(A3) holds, the first eigenvalue $\lambda_{1}$ of (3) is simple, isolated and positive, and has a unique associated eigenfunction $\left(\mu_{1}, v_{1}\right)$ with $\left\|\left(\mu_{1}, v_{1}\right)\right\|=1$ and $\mu_{1}>0$, $v_{1}>0$ in $\Omega$ (the proof is found in $[1,2]$ ).

The Landesman-Lazer-type conditions were introduced by Landesman and Lazer in [3], where they considered the existence of weak solutions for the resonant elliptic problems, and then were widely used and extended (see [1-10] and their references). For nonlinear elliptic systems, let $F_{s}(x, s, t)=g_{1}(s), F_{t}(x, s, t)=g_{2}(t)$ and by using the some LandesmanLazer-type conditions, Zographopoulos in [1] proved the existence of weak solutions for problem (1) at resonance with the first eigenvalue $\lambda_{1}$, and by using the Landesman-Lazertype conditions due to Tang and the G-linking theorem, Ou and Tang in [2] proved the existence of weak solutions for problem (1) at resonance with the higher eigenvalues of problem (3). When $p=2$, Silva in [10] introduced the new Landesman-Lazer-type conditions and proved the existence of weak solutions for problem (1) by using variational methods, Morse theory and critical groups.

Motivated by [10], we consider the existence of weak solutions for problem (1) under the certain Landesman-Lazer-type conditions. We now give some auxiliary conditions.

(F1) There is $h \in C\left(\Omega, R^{+}\right)$such that

$$
\left|F_{s}(x, s, t)\right| \leq h(x) \quad \text { and } \quad\left|F_{t}(x, s, t)\right| \leq h(x), \quad \forall(x, s, t) \in \Omega \times R^{2} .
$$

(F2) There exist functions $f^{++}, f^{--} \in C(\Omega, R)$ such that

$$
f^{++}(x)=\lim _{\substack{s \rightarrow+\infty \\ t \rightarrow+\infty}} F_{s}(x, s, t), \quad f^{--}(x)=\lim _{\substack{s \rightarrow-\infty \\ t \rightarrow-\infty}} F_{s}(x, s, t) .
$$

(F3) There exist functions $g^{++}, g^{--} \in C(\Omega, R)$ such that

$$
g^{++}(x)=\lim _{\substack{s \rightarrow+\infty \\ t \rightarrow+\infty}} F_{t}(x, s, t), \quad g^{--}(x)=\lim _{\substack{s \rightarrow+\infty \\ t \rightarrow+\infty}} F_{t}(x, s, t)
$$

where the above limits of conditions (F2) and (F3) are taken uniformly for all $x \in \Omega$. The Landesman-Lazer-type conditions for problem (1) will be assumed either

$$
(L L)_{1}^{+} \quad \int_{\Omega} f^{--} \mu_{1}+g^{--} v_{1} d x<\int_{\Omega} h_{1} \mu_{1}+h_{2} v_{1} d x<\int_{\Omega} f^{++} \mu_{1}+g^{++} v_{1} d x
$$

or

$$
(L L)_{1}^{-} \quad \int_{\Omega} f^{--} \mu_{1}+g^{--} v_{1} d x>\int_{\Omega} h_{1} \mu_{1}+h_{2} v_{1} d x>\int_{\Omega} f^{++} \mu_{1}+g^{++} v_{1} d x .
$$

We are ready to introduce the main results of this paper. 
Theorem 1 Assume that $h_{1}, h_{2} \in L^{q}(\Omega)(q=p /(p-1))$ and one of the conditions (A1)-(A3) holds. If F satisfies (F1), (F2), (F3) and (LL) , then problem (1) has at least one solution.

Theorem 2 Assume that $h_{1}, h_{2} \in L^{q}(\Omega)(q=p /(p-1))$ and one of the conditions (A1)-(A3) holds. If $F$ satisfies (F1), (F2), (F3) and (LL) $)_{1}^{-}$, then problem (1) has at least one solution.

\section{Proofs of theorems}

Let $J: W \rightarrow R$ be the functional defined by

$$
J(u, v)=\phi(u, v)-\lambda_{1} \psi(u, v)-\int_{\Omega} F(x, u, v) d x+\int_{\Omega} h_{1}(x) u d x+\int_{\Omega} h_{2}(x) v d x,
$$

where

$$
\begin{aligned}
& \phi(u, v)=\frac{1}{p} \int_{\Omega}|\nabla u|^{p} d x+\frac{1}{p} \int_{\Omega}|\nabla v|^{p} d x, \quad \text { and } \\
& \psi(u, v)=\frac{1}{p} \int_{\Omega} a(x)|u|^{p} d x+\frac{1}{p} \int_{\Omega} c(x)|v|^{p} d x+\frac{1}{(\alpha+1)(\beta+1)} \int_{\Omega} b(x)|u|^{\alpha}|v|^{\beta} u v d x .
\end{aligned}
$$

If one of the conditions (A1)-(A3) holds, by (F1) and $h_{1}, h_{2} \in L^{q}(\Omega)$, it is not difficult to verify that $J \in C^{1}(W, R)$, and it is well known that a critical point of the functional $J$ in $W$ corresponds to a weak solution of problem (1). We will prove Theorem 1 by the saddle point theorem due to Rabinowitz (see [11]) and Theorem 2 by Ekeland's variational principle (see [12]).

Proof of Theorem 1 We divide the proof into two steps.

(i) We claim that the functional $J$ satisfies the $(P S)$ condition. Let $\left(u_{n}, v_{n}\right) \in W$ be a $(P S)$ sequence for the functional $J$, that is,

$$
J\left(u_{n}, v_{n}\right) \rightarrow c \in R \quad \text { and } \quad J^{\prime}\left(u_{n}, v_{n}\right) \rightarrow 0 \quad \text { as } n \rightarrow \infty .
$$

We first verify that $\left(u_{n}, v_{n}\right)$ is bounded in $W$, and then prove that $\left(u_{n}, v_{n}\right)$ has a convergent subsequence. Suppose, by contradiction, that $K_{n}:=\left\|\left(u_{n}, v_{n}\right)\right\|=\left(\left\|u_{n}\right\|^{p}+\left\|v_{n}\right\|^{p}\right)^{1 / p} \rightarrow \infty$ as $n \rightarrow \infty$. Let $\tilde{u}_{n}=u_{n} \backslash K_{n}, \tilde{v}_{n}=v_{n} \backslash K_{n}$, then $\left(\tilde{u}_{n}, \tilde{v}_{n}\right)$ is bounded in $W$, that is,

$$
\left\|\tilde{u}_{n}\right\|^{p}+\left\|\tilde{v}_{n}\right\|^{p}=1 \quad \text { for all } n .
$$

Hence there is a subsequence of $\left(\tilde{u}_{n}, \tilde{v}_{n}\right)$, still denoted by $\left(\tilde{u}_{n}, \tilde{v}_{n}\right)$, and $(\tilde{u}, \tilde{v}) \in W$ such that $\left(\tilde{u}_{n}, \tilde{v}_{n}\right) \rightarrow(\tilde{u}, \tilde{v})$ weakly in $W,\left(\tilde{u}_{n}, \tilde{v}_{n}\right) \rightarrow(\tilde{u}, \tilde{v})$ strongly in $L^{p}(\Omega) \times L^{p}(\Omega)$ and $\left(\tilde{u}_{n}(x), \tilde{v}_{n}(x)\right) \rightarrow(\tilde{u}(x), \tilde{v}(x))$ for a.e. $x \in \Omega$. From (F1), (2) and Hölder's inequality, we obtain

$$
\begin{aligned}
\left|\int_{\Omega} F(x, u, v) d x\right| & \leq \int_{\Omega}|F(x, u, v)| d x \\
& =\int_{\Omega}|F(x, u, v)-F(x, 0,0)+F(x, 0,0)| d x \\
& \leq \int_{\Omega}\left|\int_{0}^{1}\left(F_{s}(x, \tau u, \tau v) u+F_{t}(x, \tau u, \tau v) v\right) d \tau\right| d x+\int_{\Omega}|F(x, 0,0)| d x
\end{aligned}
$$




$$
\begin{aligned}
& \leq \int_{\Omega} h(x)(|u|+|v|) d x+C_{0} \\
& \leq C\|h\|_{L^{q}}(\|u\|+\|v\|)+C_{0}
\end{aligned}
$$

for all $(u, v) \in W$, where $C_{0}=\int_{\Omega}|F(x, 0,0)| d x$, hence we get

$$
\frac{1}{\left\|u_{n}\right\|^{p}+\left\|v_{n}\right\|^{p}} \int_{\Omega} F\left(x, u_{n}, v_{n}\right) d x \rightarrow 0 \quad \text { as } n \rightarrow \infty,
$$

and from $h_{1}, h_{2} \in L^{q}(\Omega)(q=p /(p-1))$ and Hölder's inequality, it follows that

$$
\frac{1}{\left\|u_{n}\right\|^{p}+\left\|v_{n}\right\|^{p}} \int_{\Omega}\left(h_{1} u_{n}+h_{2} v_{n}\right) d x \rightarrow 0 \quad \text { as } n \rightarrow \infty .
$$

From $\left(\tilde{u}_{n}, \tilde{v}_{n}\right) \rightarrow(\tilde{u}, \tilde{v})$ strongly in $L^{p}(\Omega) \times L^{p}(\Omega)$, we have $\left|\tilde{u}_{n}\right|^{p} \rightarrow|\tilde{u}|^{p}$ and $\left|\tilde{v}_{n}\right|^{p} \rightarrow|\tilde{v}|^{p}$ strongly in $L^{1}(\Omega) \times L^{1}(\Omega)$. Hence, it follows that

$$
\left.\left|\int_{\Omega} a(x)\right| \tilde{u}_{n}\right|^{p} d x-\int_{\Omega} a(x)|\tilde{u}|^{p} d x\left|\leq\|a\|_{L^{\infty}} \int_{\Omega}\right|\left|\tilde{u}_{n}\right|^{p}-|\tilde{u}|^{p} \mid d x \rightarrow 0
$$

as $n \rightarrow \infty$.

From $\left(\tilde{u}_{n}(x), \tilde{v}_{n}(x)\right) \rightarrow(\tilde{u}(x), \tilde{v}(x))$ for a.e. $x \in \Omega$ and

$$
\begin{aligned}
& \left.\left.\int_{\Omega}|| \tilde{u}_{n}\right|^{\alpha} \tilde{u}_{n}\right|^{\frac{p}{\alpha+1}} d x=\left\|\tilde{u}_{n}\right\|_{L^{p}}^{p} \rightarrow\|\tilde{u}\|_{L^{p}}^{p}=\left.\left.\int_{\Omega}|| \tilde{u}\right|^{\alpha} \tilde{u}\right|^{\frac{p}{\alpha+1}} d x, \\
& \left.\left.\int_{\Omega}|| \tilde{v}_{n}\right|^{\beta} \tilde{v}_{n}\right|^{\frac{p}{\beta+1}} d x=\left\|\tilde{v}_{n}\right\|_{L^{p}}^{p} \rightarrow\|\tilde{v}\|_{L^{p}}^{p}=\left.\left.\int_{\Omega}|| \tilde{v}\right|^{\beta} \tilde{v}\right|^{\frac{p}{\beta+1}} d x
\end{aligned}
$$

as $n \rightarrow \infty$, it follows that $\left|\tilde{u}_{n}\right|^{\alpha} \tilde{u}_{n} \rightarrow|\tilde{u}|^{\alpha} \tilde{u}$ strongly in $L^{\frac{p}{\alpha+1}}(\Omega)$ and $\left|\tilde{v}_{n}\right|^{\beta} \tilde{v}_{n} \rightarrow|\tilde{v}|^{\beta} \tilde{v}$ strongly in $L^{\frac{p}{\beta+1}}(\Omega)$. Hence from Hölder's inequality we obtain

$$
\begin{aligned}
& \left|\int_{\Omega} b(x)\left(\left|\tilde{u}_{n}\right|^{\alpha}\left|\tilde{v}_{n}\right|^{\beta} \tilde{u}_{n} \tilde{v}_{n}-|\tilde{u}|^{\alpha}|\tilde{v}|^{\beta} \tilde{u} \tilde{v}\right) d x\right| \\
& \leq\left.\|b\|_{L^{\infty}} \int_{\Omega}|| \tilde{u}_{n}\right|^{\alpha}\left|\tilde{v}_{n}\right|^{\beta} \tilde{u}_{n} \tilde{v}_{n}-\left|\tilde{u}_{n}\right|^{\alpha}|\tilde{v}|^{\beta} \tilde{u}_{n} \tilde{v} \mid d x \\
& \quad+\left.\|b\|_{L^{\infty}} \int_{\Omega}|| \tilde{u}_{n}\right|^{\alpha}|\tilde{v}|^{\beta} \tilde{u}_{n} \tilde{v}-|\tilde{u}|^{\alpha}|\tilde{v}|^{\beta} \tilde{u} \tilde{v} \mid d x \\
& \leq\left.\|b\|_{L^{\infty}} \int_{\Omega}\left|\tilde{u}_{n}\right|^{\alpha+1} \cdot|| \tilde{v}_{n}\right|^{\beta} \tilde{v}_{n}-|\tilde{v}|^{\beta} \tilde{v} \mid d x \\
& \quad+\left.\|b\|_{L^{\infty}} \int_{\Omega}|| \tilde{u}_{n}\right|^{\alpha} \tilde{u}_{n}-|\tilde{u}|^{\alpha} \tilde{u}|\cdot||\tilde{v}|^{\beta+1} d x \\
& \leq\|b\|_{L^{\infty}}\left\|\tilde{u}_{n}\right\|_{L^{p}}^{\alpha+1}\left\|\left|\tilde{v}_{n}\right|^{\beta} \tilde{v}_{n}-|\tilde{v}|^{\beta} \tilde{v}\right\|_{L^{\beta}} \frac{p}{\beta+1} \\
& \quad+\|b\|_{L^{\infty}}\left\|\tilde{v}_{n}\right\|_{L^{p}}^{\beta+1}\left\|\left|\tilde{u}_{n}\right|^{\alpha} \tilde{u}_{n}-|\tilde{u}|^{\alpha} \tilde{u}\right\|_{L^{\alpha+1}}^{\frac{p}{\alpha+1}} \\
& \rightarrow 0 \quad \text { as } n \rightarrow \infty .
\end{aligned}
$$

From (5) it follows that

$$
\limsup _{n \rightarrow \infty} \frac{J\left(u_{n}, v_{n}\right)}{K_{n}^{p}} \leq 0
$$


Combining the above inequality with (7), (8), (9) (10) and $\alpha+\beta+2=p$, we have

$$
\begin{aligned}
& \limsup _{n \rightarrow \infty}\left(\int_{\Omega}\left|\nabla \tilde{u}_{n}\right|^{p} d x+\int_{\Omega}\left|\nabla \tilde{v}_{n}\right|^{p} d x\right) \\
& \quad \leq \lambda_{1}\left(\int_{\Omega} a(x)|\tilde{u}|^{p} d x+\int_{\Omega} c(x)|\tilde{v}|^{p} d x+\frac{p}{(\alpha+1)(\beta+1)} \int_{\Omega} b(x)|\tilde{u}|^{\alpha}|\tilde{v}|^{\beta} \tilde{u} \tilde{v} d x\right) .
\end{aligned}
$$

Hence, using the weak lower semicontinuity of the norm and the Poincaré inequality, we obtain

$$
\begin{aligned}
& \lambda_{1}\left(\int_{\Omega} a(x)|\tilde{u}|^{p} d x+\int_{\Omega} c(x)|\tilde{v}|^{p} d x+\frac{p}{(\alpha+1)(\beta+1)} \int_{\Omega} b(x)|\tilde{u}|^{\alpha}|\tilde{v}|^{\beta} \tilde{u} \tilde{v} d x\right) \\
& \quad \leq \int_{\Omega}|\nabla \tilde{u}|^{p} d x+\int_{\Omega}|\nabla \tilde{v}|^{p} d x \\
& \quad \leq \liminf _{n \rightarrow \infty}\left(\int_{\Omega}\left|\nabla \tilde{u}_{n}\right|^{p} d x+\int_{\Omega}\left|\nabla \tilde{v}_{n}\right|^{p} d x\right) \\
& \quad \leq \limsup _{n \rightarrow \infty}\left(\int_{\Omega}\left|\nabla \tilde{u}_{n}\right|^{p} d x+\int_{\Omega}\left|\nabla \tilde{v}_{n}\right|^{p} d x\right) \\
& \quad \leq \lambda_{1}\left(\int_{\Omega} a(x)|\tilde{u}|^{p} d x+\int_{\Omega} c(x)|\tilde{v}|^{p} d x+\frac{p}{(\alpha+1)(\beta+1)} \int_{\Omega} b(x)|\tilde{u}|^{\alpha}|\tilde{v}|^{\beta} \tilde{u} \tilde{v} d x\right),
\end{aligned}
$$

which implies that the following equality holds:

$$
\begin{aligned}
& \int_{\Omega}|\nabla \tilde{u}|^{p} d x+\int_{\Omega}|\nabla \tilde{v}|^{p} d x \\
& \quad=\lambda_{1}\left(\int_{\Omega} a(x)|\tilde{u}|^{p} d x+\int_{\Omega} c(x)|\tilde{v}|^{p} d x+\frac{p}{(\alpha+1)(\beta+1)} \int_{\Omega} b(x)|\tilde{u}|^{\alpha}|\tilde{v}|^{\beta} \tilde{u} \tilde{v} d x\right) .
\end{aligned}
$$

By the uniform convexity of $W$, we have that $\left(\tilde{u}_{n}, \tilde{v}_{n}\right)$ converges strongly to $(\tilde{u}, \tilde{v})$ in $W$, and from the definition of $\left(\mu_{1}, v_{1}\right)$, it follows that $(\tilde{u}, \tilde{v})= \pm\left(\mu_{1}, v_{1}\right)$.

In the following, we assume that $(\tilde{u}, \tilde{v})=\left(\mu_{1}, \nu_{1}\right)$, and the case where $(\tilde{u}, \tilde{v})=-\left(\mu_{1}, \nu_{1}\right)$ may be treated similarly. Noting that $\alpha+\beta+2=p$, it follows that

$$
\begin{aligned}
& \frac{p}{K_{n}(\alpha+1)(\beta+1)} \int_{\Omega} b(x)\left|u_{n}\right|^{\alpha}\left|v_{n}\right|^{\beta} u_{n} v_{n} d x \\
& =\frac{1}{\beta+1} \int_{\Omega} b(x)\left|u_{n}\right|^{\alpha}\left|v_{n}\right|^{\beta} \tilde{u}_{n} v_{n} d x+\frac{1}{\alpha+1} \int_{\Omega} b(x)\left|u_{n}\right|^{\alpha}\left|v_{n}\right|^{\beta} u_{n} \tilde{v}_{n} d x .
\end{aligned}
$$

Hence from (4) and the above equality, we have

$$
\begin{aligned}
& \frac{p J\left(u_{n}, v_{n}\right)}{K_{n}}-\left\langle J^{\prime}\left(u_{n}, v_{n}\right),\left(\tilde{u}_{n}, \tilde{v}_{n}\right)\right\rangle \\
& =\int_{\Omega}\left(F_{s}\left(x, u_{n}, v_{n}\right) \tilde{u}_{n}+F_{t}\left(x, u_{n}, v_{n}\right) \tilde{v}_{n}\right) d x-\frac{p}{K_{n}} \int_{\Omega} F\left(x, u_{n}, v_{n}\right) d x \\
& \quad+(p-1) \int_{\Omega}\left(h_{1} \tilde{u}_{n}+h_{2} \tilde{v}_{n}\right) d x .
\end{aligned}
$$


From $h_{1}, h_{2} \in L^{q}(\Omega)$, we observe

$$
\int_{\Omega} h_{1} \tilde{u}_{n}+h_{2} \tilde{v}_{n} d x \rightarrow \int_{\Omega} h_{1} \mu_{1}+h_{2} v_{1} d x \quad \text { as } n \rightarrow \infty
$$

From (F2) and (F3), we have

$$
\int_{\Omega} F_{s}\left(x, u_{n}, v_{n}\right) \tilde{u}_{n}+F_{t}\left(x, u_{n}, v_{n}\right) \tilde{v}_{n} d x \rightarrow \int_{\Omega}\left(f^{++} \mu_{1}+g^{++} v_{1}\right) d x \quad \text { as } n \rightarrow \infty
$$

Finally, from the Lebesgue dominated convergence theorem, (F2) and (F3), we have

$$
\begin{aligned}
\frac{1}{K_{n}} & \int_{\Omega} F\left(x, u_{n}, v_{n}\right) d x \\
& =\frac{1}{K_{n}} \int_{\Omega} \int_{0}^{1}\left(F_{s}\left(x, \tau u_{n}, \tau v_{n}\right) u_{n}+F_{t}\left(x, \tau u_{n}, \tau v_{n}\right) v_{n}\right) d \tau d x+\frac{C_{0}}{K_{n}} \\
& =\int_{\Omega} \int_{0}^{1}\left(F_{s}\left(x, \tau u_{n}, \tau v_{n}\right) \tilde{u}_{n}+F_{t}\left(x, \tau u_{n}, \tau v_{n}\right) \tilde{v}_{n}\right) d \tau d x+\frac{C_{0}}{K_{n}} \\
& \rightarrow \int_{\Omega}\left(f^{++} \mu_{1}+g^{++} v_{1}\right) d x \quad \text { as } n \rightarrow \infty .
\end{aligned}
$$

Therefore, taking the limit in (11) and from (5), (12), (13) and (14), we get

$$
\int_{\Omega}\left(h_{1} \mu_{1}+h_{2} v_{1}\right) d x=\int_{\Omega}\left(f^{++} \mu_{1}+g^{++} v_{1}\right) d x
$$

which is a contradiction with the condition $(L L)_{1}^{+}$. Hence, $\left(u_{n}, v_{n}\right)$ is bounded in $W$, and there is a subsequence of $\left(u_{n}, v_{n}\right)$ without any loss of generality still denoted by $\left(u_{n}, v_{n}\right)$, and $(u, v) \in W$ such that $\left(u_{n}, v_{n}\right) \rightarrow(u, v)$ weakly in $W,\left(u_{n}, v_{n}\right) \rightarrow(u, v)$ strongly in $L^{p}(\Omega) \times$ $L^{p}(\Omega)$. Consequently, from (5), one has

$$
\lim _{n \rightarrow \infty}\left\langle J^{\prime}\left(u_{n}, v_{n}\right),\left(u_{n}-u, 0\right)\right\rangle=0
$$

From (F1) and Hölder's inequality, it follows that

$$
\left|\int_{\Omega} F_{s}\left(x, u_{n}, v_{n}\right)\left(u_{n}-u\right) d x\right| \leq\|h\|_{L^{q}}\left\|u_{n}-u\right\|_{L^{p}} \rightarrow 0
$$

as $n \rightarrow \infty$. Similarly, we obtain

$$
\left|\int_{\Omega} h_{1}(x)\left(u_{n}-u\right) d x\right| \leq\left\|h_{1}\right\|_{L^{q}}\left\|u_{n}-u\right\|_{L^{p}} \rightarrow 0
$$

and

$$
\begin{aligned}
\left.\left|\int_{\Omega} a(x)\right| u_{n}\right|^{p-2} u_{n}\left(u_{n}-u\right) d x \mid & \leq\|a\|_{L^{\infty}}\left\|u_{n}\right\|_{L^{p}}^{p-1}\left\|u_{n}-u\right\|_{L^{p}} \\
& \leq C^{p-1}\|a\|_{L^{\infty}}\left\|u_{n}\right\|^{p-1}\left\|u_{n}-u\right\|_{L^{p}} \\
& \rightarrow 0
\end{aligned}
$$


as $n \rightarrow \infty$. Combining the above three inequalities and (15), we get

$$
\int_{\Omega}\left(\left|\nabla u_{n}\right|^{p-2} \nabla u_{n}, \nabla\left(u_{n}-u\right)\right) d x \rightarrow 0
$$

as $n \rightarrow \infty$. Similarly, we also obtain

$$
\lim _{n \rightarrow \infty} \int_{\Omega}\left(|\nabla u|^{p-2} \nabla u, \nabla\left(u_{n}-u\right)\right) d x=0
$$

hence

$$
\lim _{n \rightarrow \infty} \int_{\Omega}\left(\left(\left|\nabla u_{n}\right|^{p-2} \nabla u_{n}-|\nabla u|^{p-2} \nabla u\right), \nabla\left(u_{n}-u\right)\right) d x=0
$$

From Clarkson's inequality, that is, there is $C_{p}>0$ such that for all $\mu, v \in R^{N}$ and $p \geq 2$,

$$
|\mu-v|^{p} \leq C_{p}\left(|\mu|^{p-2} \mu-|v|^{p-2} v\right)(\mu-v)
$$

it follows that

$$
\lim _{n \rightarrow \infty} \int_{\Omega}\left|\nabla u_{n}-\nabla u\right|^{p} d x=0
$$

this is, $u_{n} \rightarrow u$ in $W_{0}^{1, p}(\Omega)$. Similarly, we have $v_{n} \rightarrow v$ in $W_{0}^{1, p}(\Omega)$, hence $\left(u_{n}, v_{n}\right) \rightarrow(u, v)$ strongly in $W$.

(ii) We claim that the functional $J$ satisfies the geometries of the saddle point theorem with respect to $\left(E_{1}, E_{2}\right)$, where $E_{1}=\operatorname{span}\left\{\left(\mu_{1}, \nu_{1}\right)\right\}, E_{2}=\left\{(\phi, \psi) \in W: \int_{\Omega}\left(\mu_{1}^{p-1} \phi+\right.\right.$ $\left.\left.\nu_{1}^{p-1} \psi\right) d x=0\right\}$ and $W=E_{1} \oplus E_{2}$.

By the definition of $\left(\mu_{1}, \nu_{1}\right)$, for all $t \in R$, we have

$$
\begin{aligned}
& \lambda_{1}\left(\int_{\Omega} a(x)\left|t \mu_{1}\right|^{p} d x+\int_{\Omega} c(x)\left|t v_{1}\right|^{p} d x\right. \\
& \left.\quad+\frac{p}{(\alpha+1)(\beta+1)} \int_{\Omega} b(x)\left|t \mu_{1}\right|^{\alpha}\left|t v_{1}\right|^{\beta} t \mu_{1} t v_{1} d x\right) \\
& \quad=\int_{\Omega}\left|\nabla\left(t \mu_{1}\right)\right|^{p} d x+\int_{\Omega}\left|\nabla\left(t v_{1}\right)\right|^{p} d x .
\end{aligned}
$$

Moreover, we have

$$
\begin{aligned}
& \int_{\Omega} F\left(x, t \mu_{1}, t v_{1}\right) d x \\
& \quad=\int_{\Omega}\left(F\left(x, t \mu_{1}, t v_{1}\right)-F(x, 0,0)\right) d x+\int_{\Omega} F(x, 0,0) d x \\
& \quad=\int_{\Omega} \int_{0}^{1}\left(F_{s}\left(x, \tau t \mu_{1}, \tau t v_{1}\right) t \mu_{1}+F_{t}\left(x, \tau t \mu_{1}, \tau t \nu_{1}\right) t \nu_{1}\right) d \tau d x+\int_{\Omega} F(x, 0,0) d x \\
& =t \int_{\Omega} \int_{0}^{1}\left(F_{s}\left(x, \tau t \mu_{1}, \tau t v_{1}\right) \mu_{1}+F_{t}\left(x, \tau t \mu_{1}, \tau t v_{1}\right) \nu_{1}\right) d \tau d x+\int_{\Omega} F(x, 0,0) d x
\end{aligned}
$$


From the Lebesgue dominated convergence theorem, (F1), (F2) and (F3), we obtain

$$
\begin{aligned}
& \lim _{t \rightarrow+\infty} \int_{\Omega} \int_{0}^{1}\left(F_{s}\left(x, \tau t \mu_{1}, \tau t v_{1}\right) \mu_{1}+F_{t}\left(x, \tau t \mu_{1}, \tau t v_{1}\right) v_{1}\right) d \tau d x \\
& \quad=\int_{\Omega}\left(f^{++} \mu_{1}+g^{++} v_{1}\right) d x .
\end{aligned}
$$

Hence, from (4), $(L L)_{1}^{+},(16),(17)$ and (18), it follows that

$$
\begin{aligned}
J\left(t \mu_{1}, t v_{1}\right) & =t \int_{\Omega}\left(h_{1} \mu_{1}+h_{2} v_{1}\right) d x-\int_{\Omega} F\left(x, t \mu_{1}, t v_{1}\right) d x \\
& \rightarrow-\infty \text { as } t \rightarrow \infty
\end{aligned}
$$

Similarly, if $t$ tends to $-\infty$, the same result is obtained with $f^{++}$and $g^{++}$exchanged with $f^{--}$and $g^{--}$respectively. Hence, in both cases we have

$$
\lim _{|t| \rightarrow \infty} J\left(t \mu_{1}, t v_{1}\right)=-\infty
$$

On the other hand, from the definition of $\lambda_{1}$, there is $\bar{\lambda}>\lambda_{1}$ such that

$$
\begin{aligned}
& \int_{\Omega}|\nabla u|^{p} d x+\int_{\Omega}|\nabla v|^{p} d x \\
& \quad \geq \bar{\lambda}\left(\int_{\Omega} a(x)|u|^{p} d x+\int_{\Omega} c(x)|v|^{p} d x+\frac{p}{(\alpha+1)(\beta+1)} \int_{\Omega} b(x)|u|^{\alpha}|v|^{\beta} u v d x\right)
\end{aligned}
$$

for all $(u, v) \in E_{2}$. From (2), (4), (6), the above inequality and Hölder's inequality, we obtain

$$
\begin{aligned}
J(u, v) \geq & \frac{\bar{\lambda}-\lambda_{1}}{p \bar{\lambda}}\left(\|u\|^{p}+\|v\|^{p}\right)-C\|h\|_{L^{q}}(\|u\|+\|v\|) \\
& -\left(\left\|h_{1}\right\|_{L^{q}}\|u\|_{L^{p}}+\left\|h_{2}\right\|_{L^{q}}\|v\|_{L^{p}}\right)-C_{0} \\
\geq & \frac{\bar{\lambda}-\lambda_{1}}{p \bar{\lambda}}\left(\|u\|^{p}+\|v\|^{p}\right)-C_{1}(\|u\|+\|v\|)-C_{0}
\end{aligned}
$$

for all $(u, v) \in E_{2}$, where $C_{1}=C\left(\|h\|_{L^{q}}+\min \left\{\left\|h_{1}\right\|_{L^{q}},\left\|h_{2}\right\|_{L^{q}}\right\}\right)$.

Thus, from (19) and (20), there is $\delta \in R$ and $R_{0}>0$ such that if $|t|=R_{0}$ we obtain

$$
J\left(t \mu_{1}, t v_{1}\right)<\delta<\min _{(u, v) \in E_{2}} J(u, v)
$$

From the saddle point theorem, Theorem 1 is proved.

Proof of Theorem 2 (i) Similar to (i) of the proof of Theorem 1, we can prove that from $(L L)_{1}^{-}$, the functional $J$ satisfies the $(P S)$ condition.

(ii) Now we will prove that the functional $J$ is coercive, that is,

$$
J(u, v) \rightarrow+\infty \quad \text { as }\|(u, v)\| \rightarrow \infty
$$


If the claim does not hold, there is a constant $c$ and a sequence $\left(u_{n}, v_{n}\right)$ with $\left\|\left(u_{n}, v_{n}\right)\right\| \rightarrow \infty$ as $n \rightarrow \infty$ such that $J\left(u_{n}, v_{n}\right) \leq c$. Let $K_{n}:=\left(\left\|u_{n}\right\|^{p}+\left\|v_{n}\right\|^{p}\right)^{1 / p}$, hence we have $K_{n} \rightarrow \infty$ as $n \rightarrow \infty$ and

$$
\limsup _{n \rightarrow \infty} \frac{J\left(u_{n}, v_{n}\right)}{K_{n}} \leq 0 .
$$

Define $\tilde{u}_{n}=u_{n} \backslash K_{n}, \tilde{v}_{n}=v_{n} \backslash K_{n}$, similar to the proof of the $(P S)$ condition of Theorem 1 again, we obtain that $\left(\tilde{u}_{n}, \tilde{v}_{n}\right)$ converges strongly to $\pm\left(\mu_{1}, v_{1}\right)$ as $n \rightarrow \infty$.

Assume that $\left(\tilde{u}_{n}, \tilde{v}_{n}\right)$ converges strongly to $\left(\mu_{1}, v_{1}\right)$ as $n \rightarrow \infty$ (the case $\left(\tilde{u}_{n}, \tilde{v}_{n}\right)$ converges strongly to $-\left(\mu_{1}, v_{1}\right)$ as $n \rightarrow \infty$ may be treated similarly), from (14) we have

$$
\begin{aligned}
0 & \geq \limsup _{n \rightarrow \infty} \frac{J\left(u_{n}, v_{n}\right)}{K_{n}} \\
& \geq \lim _{n \rightarrow \infty}\left(\int_{\Omega} h_{1} \tilde{u}_{n}+h_{2} \tilde{v}_{n} d x-\frac{1}{K_{n}} \int_{\Omega} F\left(x, u_{n}, v_{n}\right) d x\right) \\
& =\int_{\Omega}\left(h_{1} \mu_{1}+h_{2} v_{1}\right) d x-\int_{\Omega}\left(f^{++} \mu_{1}+g^{++} v_{1}\right) d x,
\end{aligned}
$$

which is a contradiction with $(L L)_{1}^{-}$. By Ekeland's variational principle, Theorem 2 is proved.

\section{Competing interests}

The authors declare that there is no conflict of interests regarding the publication of this article.

\section{Authors' contributions}

All authors typed, read and approved the final manuscript.

\section{Acknowledgements}

The work was in part supported by the National Natural Science Foundation of China (No. 11101347) and the Fundamental Research Funds for the Central Universities (No. XDJK2011C039), the Postdoctoral Research Foundation of Chongqing (No. Xm201319). The authors would like to thank an anonymous referee and the editor for the helpful comments.

Received: 26 March 2015 Accepted: 9 October 2015 Published online: 26 October 2015

\section{References}

1. Zographopoulos, NB: p-Laplacian systems on resonance. Appl. Anal. 83(5), 509-519 (2004)

2. Ou, Z-Q, Tang, C-L: Resonance problems for the $p$-Laplacian systems. J. Math. Anal. Appl. 345, 511-521 (2008)

3. Landesman, M, Lazer, A-C: Nonlinear perturbations of linear elliptic boundary value problems at resonance. J. Math. Mech. 19, 609-623 (1970)

4. Arcoya, D, Orsina, L: Landesman-Lazer conditions and quasilinear elliptic equations. Nonlinear Anal. 28(10), 1623-1632 (1997)

5. Drábek, P, Robinson, SB: Resonance problems for the p-Laplacian. J. Funct. Anal. 169(1), 189-200 (1999)

6. Tang, C-L: Solvability for two-point boundary value problems. J. Math. Anal. Appl. 216(1), 368-374 (1997)

7. Tang, C-L: Solvability of the forced Duffing equation at resonance. J. Math. Anal. Appl. 219(1), 110-124 (1998)

8. Tang, C-L: Solvability of Neumann problem for elliptic equations at resonance. Nonlinear Anal. 44(3), 323-335 (2001)

9. Song, S-Z, Tang, C-L: Resonance problems for the $p$-Laplacian with a nonlinear boundary condition. Nonlinear Anal. 64(9), 2007-2021 (2006)

10. Da Silva, ED: Multiplicity of solutions for gradient systems using Landesman-Lazer conditions. Abstr. Appl. Anal. 2010, Article ID 237826 (2010)

11. Rabinowitz, PH: Minimax Methods in Critical Point Theory with Applications to Differential Equations. CBMS Regional Conference Series in Mathematics, vol. 65. Am. Math. Soc., Providence (1986)

12. Struwe, M: Variational Methods: Applications to Nonlinear Partial Differential Equations and Hamiltonian Systems. Springer, New York (1990) 